\title{
The Estimates of Diagonally Dominant Degree and Eigenvalue Inclusion Regions for the Schur Complement of Matrices
}

\author{
Dongjie Gao \\ Department of Mathematics, Heze University, Shandong, China \\ Email: aizai 2004@126.com
}

Received 29 July 2015; accepted 23 August 2015; published 26 August 2015

Copyright (C) 2015 by author and Scientific Research Publishing Inc.

This work is licensed under the Creative Commons Attribution International License (CC BY).

http://creativecommons.org/licenses/by/4.0/

(c) (i) 0 pen Access

\section{Abstract}

The theory of Schur complement plays an important role in many fields such as matrix theory, control theory and computational mathematics. In this paper, some new estimates of diagonally, $\alpha$-diagonally and product $\alpha$-diagonally dominant degree on the Schur complement of matrices are obtained, which improve some relative results. As an application, we present several new eigenvalue inclusion regions for the Schur complement of matrices. Finally, we give a numerical example to illustrate the advantages of our derived results.

\section{Keywords}

Schur Complement, Gerschgorin Theorem, Diagonally Dominant Degree, Eigenvalue

\section{Introduction}

Let $\mathbb{C}^{n \times n}$ denote the set of all $n \times n$ complex matrices, $N=\{1,2, \cdots, n\}$ and $\boldsymbol{A}=\left(a_{i j}\right) \in \mathbb{C}^{n \times n}(n \geq 2)$. We write

$$
\begin{gathered}
R_{i}(\boldsymbol{A})=\sum_{j \neq i}\left|a_{i j}\right|, C_{i}(\boldsymbol{A})=\sum_{j \neq i}\left|a_{j i}\right|, i \in N, \\
N_{r}(\boldsymbol{A})=\left\{i|| a_{i i} \mid>R_{i}(\boldsymbol{A}), i \in N\right\}, N_{c}(\boldsymbol{A})=\left\{i|| a_{i i} \mid>C_{i}(\boldsymbol{A}), i \in N\right\} .
\end{gathered}
$$

We know that $\boldsymbol{A}$ is called a strictly diagonally dominant matrix if

$$
\left|a_{i i}\right|>R_{i}(\boldsymbol{A}), \forall i \in N \text {. }
$$

$\boldsymbol{A}$ is called an Ostrowski matrix (see [1]) if 


$$
\left|a_{i i}\right|\left|a_{j j}\right|>R_{i}(A) R_{j}(A), \forall i, j \in N, i \neq j .
$$

$S D_{n}$ and $O S_{n}$ will be used to denote the sets of all $n \times n$ strictly diagonally dominant matrices and the sets all $n \times n$ Ostrowski matrices, respectively.

As shown in [2], for $1 \leq i \leq n$ and $\alpha \in[0,1]$, we call $\left|a_{i i}\right|-R_{i}(\boldsymbol{A}),\left|a_{i i}\right|-\alpha R_{i}(\boldsymbol{A})-(1-\alpha) C_{i}(\boldsymbol{A})$ and $\left|a_{i i}\right|-\left[R_{i}(\boldsymbol{A})\right]^{\alpha}\left[C_{i}(\boldsymbol{A})\right]^{1-\alpha}$ the $i$-th diagonally, $\alpha$-diagonally and product $\alpha$-diagonally dominant degree of $\boldsymbol{A}$, respectively.

For $\beta \subseteq N$, denote by $|\beta|$ the cardinality of $\beta$ and $\bar{\beta}=N / \beta$. If $\beta, \gamma \subseteq N$, then $\boldsymbol{A}(\beta, \gamma)$ is the submatrix of $\boldsymbol{A}$ with row indices in $\beta$ and column indices in $\gamma$. In particular, $\boldsymbol{A}(\beta, \beta)$ is abbreviated to $\boldsymbol{A}(\beta)$. If $\boldsymbol{A}(\beta)$ is nonsingular,

$$
\boldsymbol{A} / \beta=\boldsymbol{A} / \boldsymbol{A}(\beta)=\boldsymbol{A}(\bar{\beta})-\boldsymbol{A}(\bar{\beta}, \beta)[\boldsymbol{A}(\beta)]^{-1} \boldsymbol{A}(\beta, \bar{\beta}),
$$

is called the Schur complement of $\boldsymbol{A}$ with respect to $\boldsymbol{A}(\beta)$.

The comparison matrix of $\boldsymbol{A}, \mu(\boldsymbol{A})=\left(\alpha_{i j}\right)$, is defined by

$$
\alpha_{i j}= \begin{cases}\left|a_{i j}\right|, & \text { if } i=j, \\ -\left|a_{i j}\right|, & \text { if } i \neq j .\end{cases}
$$

A matrix $\boldsymbol{A}=\left(a_{i j}\right) \in \mathbb{C}^{n \times n}$ is called an $M$-matrix, if there exist a nonnegative matrix $B$ and a real number $s>\rho(\boldsymbol{B})$, where $\rho(\boldsymbol{B})$ is the spectral radius of $\boldsymbol{B}$, such that $\boldsymbol{A}=s \boldsymbol{I}-\boldsymbol{B}$. It is known that $A$ is an $h$-matrix if and only if $\mu(\boldsymbol{A})$ is an $m$-matrix, and if $A$ is an $m$-matrix, then the Schur complement of $A$ is also an $m$-matrix and det $\boldsymbol{A}>0$ (see [3]). We denote by $H_{n}$ and $M_{n}$ the sets of $h$-matrices and $m$-matrices, respectively.

The Schur complement of matrix is an important part of matrix theory, which has been proved to be useful tools in many fields such as control theory, statistics and computational mathematics. A lot of work has been done on it (see [4]-[8]). We know that the Schur complements of strictly diagonally dominant matrices are strictly diagonally dominant matrices, and the Schur complements of Ostrowski matrices are Ostrowski matrices. These properties have been used for deriving matrix inequalities in matrix analysis and for the convergence of iterations in numerical analysis (see [9]-[12]). More importantly, studying the locations for the eigenvalues of the Schur complement is of great significance, as shown in [2] [6] [13]-[18].

The paper is organized as follows. In Section 2, we give some new estimates of diagonally dominant degree on the Schur complement of matrices. In Section 3, we present several new eigenvalue inclusion regions for the Schur complement of matrices. In Section 4, we give a numerical example to illustrate the advantages of our derived results.

\section{The Diagonally Dominant Degree for the Schur Complement}

In this section, we present several new estimates of diagonally, $\alpha$-diagonally and product $\alpha$-diagonally dominant degree on the Schur complement of matrices.

Lemma 1. [3] If $\boldsymbol{A} \in H_{n}$, then $[\mu(\boldsymbol{A})]^{-1} \geq\left|\boldsymbol{A}^{-1}\right|$.

Lemma 2. [3] If $\boldsymbol{A} \in S D_{n}$ or $\boldsymbol{A} \in O S_{n}$, then $\boldsymbol{A} \in H_{n}$, i.e., $\mu(\boldsymbol{A}) \in M_{n}$.

Lemma 3. [6] If $\boldsymbol{A} \in S D_{n}$ or $\boldsymbol{A} \in O S_{n}$ and $\beta \subseteq N$, then the Schur complement of $\boldsymbol{A}$ is in $S D_{|\bar{\beta}|}$ or $O S_{|\bar{\beta}|}$, where $\bar{\beta}=N-\beta$ is the complement of $\beta$ in $N$ and $|\bar{\beta}|$ is the cardinality of $\bar{\beta}$.

Lemma 4. [16] Let $a>b, c>b, b>0$ and $0 \leq \alpha \leq 1$. Then

$$
a^{\alpha} c^{1-\alpha} \geq(a-b)^{\alpha}(c-b)^{1-\alpha}+b \text {. }
$$

Theorem 1. Let $\boldsymbol{A}=\left(a_{i j}\right) \in \mathbb{C}^{n \times n}, \quad \beta=\left\{i_{1}, i_{2}, \cdots, i_{k}\right\} \subseteq N_{r}(\boldsymbol{A}) \neq \phi, \quad \bar{\beta}=\left\{j_{1}, j_{2}, \cdots, j_{l}\right\}, \quad 1 \leq k<n \quad$ and $A / \beta=\left(a_{t s}^{\prime}\right)$. Then for all $1 \leq t \leq l$,

$$
\left|a_{t t}^{\prime}\right|-R_{t}(\boldsymbol{A} / \beta) \geq\left|a_{j_{t} j_{t}}\right|-R_{j_{t}}(\boldsymbol{A})+\delta_{j_{t}} \geq\left|a_{j_{t} j_{t}}\right|-R_{j_{t}}(\boldsymbol{A})
$$

and

$$
\left|a_{t t}^{\prime}\right|+R_{t}(\boldsymbol{A} / \beta) \leq\left|a_{j_{t} j_{t}}\right|+R_{j_{t}}(\boldsymbol{A})-\delta_{j_{t}} \leq\left|a_{j_{t} j_{t}}\right|+R_{j_{t}}(\boldsymbol{A})
$$


where

$$
\begin{gathered}
\delta_{j_{t}}=\sum_{v=1}^{k}\left|a_{j_{l_{i}}}\right| \frac{\left|a_{i_{v} i_{v}}\right|-P_{i_{v}}(\boldsymbol{A})}{\left|a_{i_{v} i_{v}}\right|}, r=\max _{1 \leq v \leq k} \frac{\left|a_{i_{v} i_{v}}\right| \sum_{u=1}^{l}\left|a_{i_{v} j_{u}}\right|}{\left(\left|a_{i_{v} i_{v}}\right|-\sum_{u=1, \neq v}^{k}\left|a_{i_{v} i_{u}}\right|\right) R_{i_{v}}}, \\
P_{i_{v}}(\boldsymbol{A})=r R_{i_{v}}, 1 \leq v \leq k .
\end{gathered}
$$

Proof. Since $\beta \subseteq N_{r}(\boldsymbol{A}) \neq \phi$, then $\boldsymbol{A}(\beta) \in H_{k}$ and $\mu(\boldsymbol{A}(\beta)) \in M_{k}$. From Lemma 1 and Lemma 2, we have

$$
[\mu(\boldsymbol{A}(\beta))]^{-1} \geq\left|[\boldsymbol{A}(\beta)]^{-1}\right|
$$

Thus, for any $\varepsilon>0$ and $1 \leq t \leq l$, we obtain

$$
\begin{aligned}
& \left|a_{t t}^{\prime}\right|-R_{t}(A / \beta) \\
& =\left|a_{j_{t} j_{t}}-\left(a_{j_{t_{1}} i_{1}}, \cdots, a_{j_{t_{i}} i_{k}}\right)[\boldsymbol{A}(\beta)]^{-1}\left(\begin{array}{c}
a_{i_{1} j_{t}} \\
\vdots \\
a_{i_{k} j_{t}}
\end{array}\right)\right|-\sum_{s \neq t}^{l}\left|a_{j_{t} j_{s}}-\left(a_{j_{t_{1}} i_{1}}, \cdots, a_{j_{t} i_{k}}\right)[\boldsymbol{A}(\beta)]^{-1}\left(\begin{array}{c}
a_{i_{1} j_{s}} \\
\vdots \\
a_{i_{k} j_{s}}
\end{array}\right)\right| \\
& \geq\left|a_{j_{t} j_{t}}\right|-\sum_{s \neq t}^{l}\left|a_{j_{t} j_{s}}\right|-\sum_{s=1}^{l}\left(\left|a_{j_{t_{1}}}\right|, \cdots,\left|a_{j_{t} i_{k}}\right|\right)[\mu(A(\beta))]^{-1}\left(\begin{array}{c}
\left|a_{i_{1} j_{s}}\right| \\
\vdots \\
\left|a_{i_{k} j_{s}}\right|
\end{array}\right) \\
& =\left|a_{j_{t} j_{t}}\right|-R_{j_{t}}(\boldsymbol{A})+\sum_{v=1}^{k}\left|a_{j_{t} i_{v}}\right|+\left(\delta_{j_{t}}-\varepsilon\right)-\left(\delta_{j_{t}}-\varepsilon\right)-\sum_{s=1}^{l}\left(\left|a_{j_{t} i_{1}}\right|, \cdots,\left|a_{j_{t} i_{k}}\right|\right)[\mu(\boldsymbol{A}(\beta))]^{-1}\left(\begin{array}{c}
\left|a_{i_{i_{1}} j_{s}}\right| \\
\vdots \\
\left|a_{i_{k} j_{s}}\right|
\end{array}\right)
\end{aligned}
$$

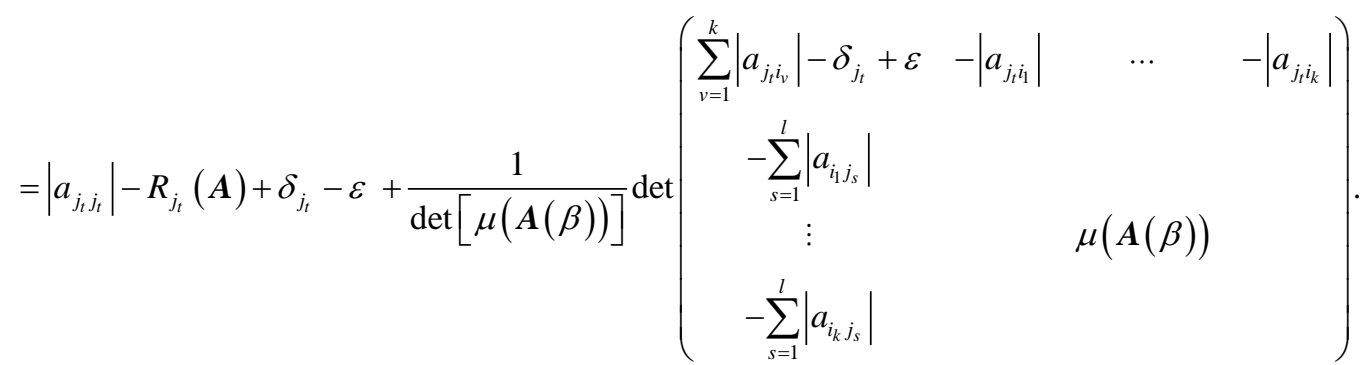

For any $j_{t} \in \bar{\beta}$, denote

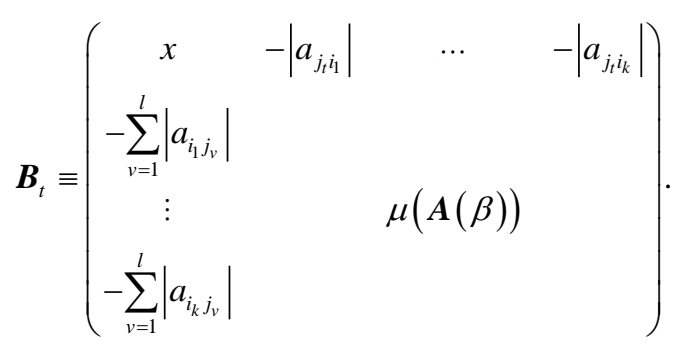

If

$$
x>\sum_{v=1}^{k}\left|a_{j t_{t} i_{v}}\right| \frac{P_{i_{v}}(\boldsymbol{A})}{\left|a_{i_{v} i_{v}}\right|},
$$


then there exists sufficiently small positive number $\varepsilon_{0}$ such that

$$
x>\sum_{v=1}^{k}\left|a_{j_{t} i_{v}}\right|\left(\frac{P_{i_{v}}(\boldsymbol{A})}{\left|a_{i_{v} i_{v}}\right|}+\varepsilon_{0}\right) .
$$

Construct a positive diagonal matrix $\boldsymbol{X}=\operatorname{diag}\left(x_{1}, x_{2}, \cdots, x_{k+1}\right)$, where

$$
x_{t}= \begin{cases}1, & \text { if } t=1 \\ \frac{P_{i_{t-1}}(A)}{\left|a_{i_{t-1} i_{t-1}}\right|}+\varepsilon_{0}, & \text { if } t=2,3, \cdots, k+1 .\end{cases}
$$

Let $\tilde{\boldsymbol{B}}=\boldsymbol{B}_{t} X=\left(\tilde{b}_{p q}\right)$. For $p=1$, by (3), we have

$$
\left|\tilde{b}_{p p}\right|-R_{p}(\tilde{\boldsymbol{B}})=\left|\tilde{b}_{11}\right|-\sum_{j=2}^{k+1}\left|\tilde{b}_{1 j}\right|=x-\sum_{v=1}^{k} \mid a_{j_{t} i_{v}}\left(\frac{P_{i_{v}}(\boldsymbol{A})}{\left|a_{i_{v} i_{v}}\right|}+\varepsilon_{0}\right)>0 .
$$

And for $p=2,3, \cdots, k+1$, by $\frac{P_{i_{v}}(\boldsymbol{A})}{\left|a_{i_{v} i_{v}}\right|} \leq r, 1 \leq v \leq k$, we obtain

$$
\begin{aligned}
& \left|\tilde{b}_{p p}\right|-R_{p}(\tilde{\boldsymbol{B}}) \\
& =\left|a_{i_{p-1} i_{p-1}}\right|\left(\frac{P_{i_{p-1}}(\boldsymbol{A})}{\left|a_{i_{p-1} i_{p-1}}\right|}+\varepsilon_{0}\right)-\sum_{v \neq p-1}^{k}\left|a_{i_{p-1} i_{v}}\right|\left(\frac{P_{i_{v}}(\boldsymbol{A})}{\left|a_{i_{v} i_{v}}\right|}+\varepsilon_{0}\right)-\sum_{v=1}^{l}\left|a_{i_{p-1} j_{v}}\right| \\
& =P_{i_{p-1}}(\boldsymbol{A})+\varepsilon_{0}\left(\left|a_{i_{p-1} i_{p-1}}\right|-\sum_{v \neq p-1}^{k}\left|a_{i_{p-1} i_{v}}\right|\right)-\sum_{v \neq p-1}^{k}\left|a_{i_{p-1} i_{v}}\right| \frac{P_{i_{v}}(\boldsymbol{A})}{\left|a_{i_{v} i_{v}}\right|}-\sum_{v=1}^{l}\left|a_{i_{p-1} j_{v}}\right| \\
& =\varepsilon_{0}\left(\left|a_{i_{p-1} i_{p-1}}\right|-\sum_{v \neq p-1}^{k}\left|a_{i_{p-1} i_{v}}\right|\right)+r \sum_{v \neq p-1}^{k}\left|a_{i_{p-1} i_{v}}\right|-\sum_{v \neq p-1}^{k}\left|a_{i_{p-1} i_{v}}\right| \frac{P_{i_{v}}(\boldsymbol{A})}{\left|a_{i_{v} i_{v}}\right|}>0 .
\end{aligned}
$$

Thus, $\tilde{\boldsymbol{B}} \in S D_{k+1}$, and so $\boldsymbol{B}_{t} \in H_{k+1}$. Note that $B_{t}=\mu\left(\boldsymbol{B}_{t}\right) \in M_{k+1}$, then

$$
\operatorname{det} \boldsymbol{B}_{t}>0 \text {. }
$$

Let $x$ be $\sum_{v=1}^{k}\left|a_{j_{t} i_{v}}\right|-\delta_{j_{t}}+\varepsilon$ in $\boldsymbol{B}_{t}$. Then

$$
\begin{aligned}
& \sum_{v=1}^{k}\left|a_{j t_{v}}\right|-\delta_{j_{t}}+\varepsilon-\sum_{v=1}^{k}\left|a_{j t_{i}}\right| \frac{P_{i_{v}}(\boldsymbol{A})}{\left|a_{i_{v} i_{v}}\right|} \\
& =\sum_{v=1}^{k}\left|a_{j t_{i} i_{v}}\right|-\sum_{v=1}^{k}\left|a_{j t_{v}}\right| \frac{\left|a_{i_{v} i_{v}}\right|-P_{i_{v}}(\boldsymbol{A})}{\left|a_{i_{v} i_{v}}\right|}-\sum_{v=1}^{k}\left|a_{j i_{v}}\right| \frac{P_{i_{v}}(\boldsymbol{A})}{\left|a_{i_{v} i_{v}}\right|}+\varepsilon>0 .
\end{aligned}
$$

Since $\operatorname{det}[\mu(A(\beta))]>0$, by (4), we have

$$
\left|a_{t t}^{\prime}\right|-R_{t}(A / \beta)>\left|a_{j_{t} j_{t}}\right|-R_{j_{t}}(A)+\delta_{j_{t}}-\varepsilon
$$

Let $\varepsilon \rightarrow 0$. Then we obtain (1). Similarly, we can prove (2).

Remark 1. Note that

$$
P_{i_{v}}(A) \leq R_{i_{v}}(A), 1 \leq v \leq k .
$$

This shows that Theorem 1 improves Theorem 2 of [17] and [2], respectively.

Next, we present some new estimates of $\alpha$-diagonally and product $\alpha$-diagonally dominant degree of the Schur complement. 
Theorem 2. Let $\boldsymbol{A}=\left(a_{i j}\right) \in \mathbb{C}^{n \times n}, \quad \beta=\left\{i_{1}, i_{2}, \cdots, i_{k}\right\} \subseteq N_{r}(\boldsymbol{A}) \cap N_{c}(\boldsymbol{A}) \neq \phi, \quad \bar{\beta}=\left\{j_{1}, j_{2}, \cdots, j_{l}\right\}, \quad 1 \leq k<n$ and $\boldsymbol{A} / \beta=\left(a_{t s}^{\prime}\right)$. Then for all $1 \leq t \leq l, \quad 0 \leq \alpha \leq 1$,

$$
\left|a_{t t}^{\prime}\right|-\left(R_{t}(\boldsymbol{A} / \beta)\right)^{\alpha}\left(C_{t}(\boldsymbol{A} / \beta)\right)^{1-\alpha} \geq\left|a_{j_{t} j_{t}}\right|-\left(R_{j_{t}}(\boldsymbol{A})-\delta_{t}\right)^{\alpha}\left(C_{j_{t}}(\boldsymbol{A})-\delta_{t}^{T}\right)^{1-\alpha},
$$

and

$$
\left|a_{t t}^{\prime}\right|+\left(R_{t}(\boldsymbol{A} / \beta)\right)^{\alpha}\left(C_{t}(\boldsymbol{A} / \beta)\right)^{1-\alpha} \leq\left|a_{j_{t} j_{t}}\right|+\left(R_{j_{t}}(\boldsymbol{A})-\delta_{t}\right)^{\alpha}\left(C_{j_{t}}(\boldsymbol{A})-\delta_{t}^{T}\right)^{1-\alpha},
$$

where for any $1 \leq v \leq k$,

$$
\begin{aligned}
& \delta_{t}=\sum_{v=1}^{k}\left|a_{j i_{v}}\right| \frac{\left|a_{i_{v} i_{v}}\right|-P_{i_{v}}(\boldsymbol{A})}{\left|a_{i_{v} i_{v}}\right|}, \eta=\max _{1 \leq \omega \leq k} \frac{\left|a_{i_{\omega} i_{\omega}}\right| \sum_{v=1}^{l}\left|a_{i_{\omega} j_{v}}\right|}{\left(\left|a_{i_{\omega} i_{\omega}}\right|-\sum_{v \neq \omega}^{l}\left|a_{i_{\omega} i_{v}}\right|\right) R_{i_{\omega}}(\boldsymbol{A})}, \\
& \delta_{t}^{T}=\sum_{v=1}^{k}\left|a_{i_{v} j_{t}}\right| \frac{\left|a_{i_{\nu} i_{v}}\right|-Q_{i_{v}}(\boldsymbol{A})}{\left|a_{i_{\nu} i_{v}}\right|}, \xi=\max _{1 \leq \omega \leq k} \frac{\left|a_{i_{\omega} i_{\omega}}\right| \sum_{v=1}^{l}\left|a_{j_{\nu} i_{\omega}}\right|}{\left(\left|a_{i_{\omega} i_{\omega}}\right|-\sum_{\nu \neq \omega}^{l}\left|a_{i_{\nu} i_{\omega}}\right|\right) C_{i_{\omega}}(\boldsymbol{A})}, \\
& P_{i_{v}}(\boldsymbol{A})=\eta R_{i_{v}}(\boldsymbol{A}), Q_{i_{v}}(\boldsymbol{A})=\xi C_{i_{v}}(\boldsymbol{A}) .
\end{aligned}
$$

Proof. By Lemma 1 and Lemma 2, we have $[\mu(\boldsymbol{A}(\beta))]^{-1} \geq\left|[\boldsymbol{A}(\beta)]^{-1}\right|$. Thus, for all $1 \leq t \leq l, 0 \leq \alpha \leq 1$, we have

$$
\begin{aligned}
& \left|a_{t t}^{\prime}\right|-\left(R_{t}(\boldsymbol{A} / \beta)\right)^{\alpha}\left(C_{t}(\boldsymbol{A} / \beta)\right)^{1-\alpha} \\
& =\left|a_{j_{t} j_{t}}-\left(a_{j_{t} i_{1}}, \cdots, a_{j_{t} i_{k}}\right)[\mathbf{A}(\beta)]^{-1}\left(\begin{array}{c}
a_{i_{1} j_{t}} \\
\vdots \\
a_{i_{k} j_{t}}
\end{array}\right)\right|-\left[\sum_{s \neq t}^{l}\left|a_{j_{t} j_{s}}+\left(a_{j_{t} i_{1}}, \cdots, a_{j_{t} i_{k}}\right)[\mathbf{A}(\beta)]^{-1}\left(\begin{array}{c}
a_{i_{1} j_{s}} \\
\vdots \\
a_{i_{k} j_{s}}
\end{array}\right)\right|\right]^{\alpha} \\
& \times\left[\sum_{s \neq t}^{l}\left|a_{j_{s} j_{t}}+\left(a_{j_{s} i_{1}}, \cdots, a_{j_{s} i_{k}}\right)[\boldsymbol{A}(\beta)]^{-1}\left(\begin{array}{c}
a_{i_{1} j_{t}} \\
\vdots \\
a_{i_{k} j_{t}}
\end{array}\right)\right|\right]^{1-\alpha} \\
& \geq\left|a_{j_{t} j_{t}}\right|-\left(\left|a_{j_{t_{1}}}\right|, \cdots,\left|a_{j_{t_{k}} i_{k}}\right|\right)[\mu(\boldsymbol{A}(\beta))]^{-1}\left(\begin{array}{c}
\left|a_{i_{1} j_{t}}\right| \\
\vdots \\
\left|a_{i_{k} j_{t}}\right|
\end{array}\right)-\left(\sum_{s \neq t}^{l}\left[\left|a_{j_{t} j_{s}}\right|+\left(\left|a_{j_{t_{1}}}\right|, \cdots,\left|a_{j_{t_{k}} i_{k}}\right|\right)[\mu(\boldsymbol{A}(\beta))]^{-1}\left(\begin{array}{c}
\left|a_{i_{1} j_{s}}\right| \\
\vdots \\
\left.\left|a_{i_{k} j_{s}}\right|\right)
\end{array}\right]\right)\right)^{\alpha} \\
& \times\left(\sum_{s \neq t}^{l}\left[\left|a_{j_{s} j_{t}}\right|+\left(\left|a_{j_{s i_{1}}}\right|, \cdots,\left|a_{j_{s} i_{k}}\right|\right)[\mu(A(\beta))]^{-1}\left(\begin{array}{c}
\left|a_{i_{1} j_{t}}\right| \\
\vdots \\
\left|a_{i_{k} j_{t}}\right|
\end{array} \mid\right)\right)^{1-\alpha}\right. \text {. }
\end{aligned}
$$

Let

$$
\zeta=\left(\left|a_{j t_{1}}\right|, \cdots,\left|a_{j i_{k}}\right|\right)[\mu(\boldsymbol{A}(\beta))]^{-1}\left(\begin{array}{c}
\left|a_{i_{1} j_{t}}\right| \\
\vdots \\
\left|a_{i_{k} j_{t}}\right|
\end{array}\right) .
$$

Similar as the proof of Theorem 1, we can prove 


$$
\sum_{s \neq t}^{l}\left[\left|a_{j_{t} j_{s}}\right|+\left(\left|a_{j i_{t} i_{1}}\right|, \cdots,\left|a_{j_{t} i_{k}}\right|\right)[\mu(\boldsymbol{A}(\beta))]^{-1}\left(\begin{array}{c}
\left|a_{i_{1} j_{s}}\right| \\
\vdots \\
\left|a_{i_{k} j_{s}}\right|
\end{array}\right] \leq R_{j_{t}}(\boldsymbol{A})-\delta_{t}-\zeta .\right.
$$

Similarly, we have

$$
\sum_{s \neq t}^{l}\left[\left|a_{j_{s} j_{t}}\right|+\left(\left|a_{j_{s} i_{1}}\right|, \cdots,\left|a_{j_{s} i_{k}}\right|\right)[\mu(\boldsymbol{A}(\beta))]^{-1}\left(\begin{array}{c}
\left|a_{i_{1} j_{t}}\right| \\
\vdots \\
\left|a_{i_{k} j_{t}}\right|
\end{array} \mid\right) \leq C_{j_{t}}(\boldsymbol{A})-\delta_{t}^{T}-\zeta .\right.
$$

By Lemma 4, we have

$$
\begin{aligned}
& \left|a_{t t}^{\prime}\right|-\left(R_{t}(\boldsymbol{A} / \beta)\right)^{\alpha}(C(\mathbf{A} / \beta))^{1-\alpha} \\
& \geq\left|a_{j_{t} j_{t}}\right|-\zeta-\left(R_{j_{t}}(\boldsymbol{A})-\delta_{t}-\zeta\right)^{\alpha}\left(C_{j_{t}}(\boldsymbol{A})-\delta_{t}^{T}-\zeta\right)^{1-\alpha} \\
& \geq\left|a_{j_{t} j_{t}}\right|-\zeta-\left[\left(R_{j_{t}}(\boldsymbol{A})-\delta_{t}\right)^{\alpha}\left(C_{j_{t}}(\boldsymbol{A})-\delta_{t}^{T}\right)^{1-\alpha}-\zeta\right] \\
& =\left|a_{j_{t} j_{t}}\right|-\left(R_{j_{t}}(\boldsymbol{A})-\delta_{t}\right)^{\alpha}\left(C_{j_{t}}(\boldsymbol{A})-\delta_{t}^{T}\right)^{1-\alpha} .
\end{aligned}
$$

Hence, (5) holds. Similarly, we can prove (6).

Remark 2. Note that

$$
P_{i_{v}}(A) \leq R_{i_{v}}(A), \quad Q_{i_{v}}(A) \leq C_{i_{v}}(A) .
$$

This shows that Theorem 3 improves Theorem 4 of [2].

Similar as the proof of Theorem 2, we can prove the following theorem immediately, which improves Theorem 2 of [2].

Theorem 3. Let $\boldsymbol{A}=\left(a_{i j}\right) \in \mathbb{C}^{n \times n}, \quad \beta=\left\{i_{1}, i_{2}, \cdots, i_{k}\right\} \subseteq N_{r}(A) \cap N_{c}(A) \neq \phi, \quad \bar{\beta}=\left\{j_{1}, j_{2}, \cdots, j_{l}\right\}, \quad 1 \leq k<n$ and $\boldsymbol{A} / \beta=\left(a_{t s}^{\prime}\right)$. Then for all $1 \leq t \leq l, 0 \leq \alpha \leq 1$,

$$
\begin{aligned}
& \left|a_{t t}^{\prime}\right|-\alpha R_{t}(\mathbf{A} / \beta)-(1-\alpha) C_{t}(\mathbf{A} / \beta) \\
& \geq\left|a_{j_{t} j_{t}}\right|-\alpha R_{j_{t}}(\mathbf{A})-(1-\alpha) C_{j_{t}}(\boldsymbol{A})+\alpha \delta_{t}+(1-\alpha) \delta_{t}^{T} \\
& \geq\left|a_{j_{t} j_{t}}\right|-\alpha R_{j_{t}}(\mathbf{A})-(1-\alpha) C_{j_{t}}(\boldsymbol{A}),
\end{aligned}
$$

and

$$
\begin{aligned}
& \left|a_{t t}^{\prime}\right|+\alpha R_{t}(\mathbf{A} / \beta)+(1-\alpha) C_{t}(\boldsymbol{A} / \beta) \\
& \leq\left|a_{j_{t} j_{t}}\right|+\alpha R_{j_{t}}(\mathbf{A})+(1-\alpha) C_{j_{t}}(\boldsymbol{A})-\alpha \delta_{t}-(1-\alpha) \delta_{t}^{T} \\
& \leq\left|a_{j_{t} j_{t}}\right|+\alpha R_{j_{t}}(\boldsymbol{A})+(1-\alpha) C_{j_{t}}(\boldsymbol{A}) .
\end{aligned}
$$

\section{Eigenvalue Inclusion Regions of the Schur Complement}

In this section, based on these derived results in Section 2, we present new eigenvalue inclusion regions for the Schur complement of matrices.

Theorem 4. Let $\boldsymbol{A}=\left(a_{i j}\right) \in \mathbb{C}^{n \times n}, \quad \beta=\left\{i_{1}, i_{2}, \cdots, i_{k}\right\} \subseteq N_{r}(\boldsymbol{A}) \neq \phi, \bar{\beta}=\left\{j_{1}, j_{2}, \cdots, j_{l}\right\}, \quad 1 \leq k<n$ and $\boldsymbol{A} / \beta=\left(a_{t s}^{\prime}\right)$ and $\lambda$ be eigenvalue of $\boldsymbol{A} / \beta$. Then there exists $1 \leq t \leq l$ such that

$$
\left|\lambda-a_{j_{t} j_{t}}\right| \leq R_{j_{t}}(A)-\delta_{j_{t}} \leq R_{j_{t}}(A) .
$$

Proof. By Gerschgorin Circle Theorem, we know that there exists $1 \leq t \leq l$ such that $\left|\lambda-a_{t t}^{\prime}\right| \leq R_{t}(\boldsymbol{A} / \beta)$. Thus, by Lemma 1 and Lemma 2, we have 


$$
\begin{aligned}
& 0 \geq\left|\lambda-a_{t t}^{\prime}\right|-R_{t}(A / \beta) \\
& =\left|\lambda-a_{j_{t} j_{t}}+\left(a_{j_{t} i_{1}}, \cdots, a_{j_{t} i_{k}}\right)[\boldsymbol{A}(\beta)]^{-1}\left(\begin{array}{c}
a_{i_{1} j_{t}} \\
\vdots \\
a_{i_{k} j_{t}}
\end{array}\right)\right|-\sum_{s=1, \neq t}^{l}\left|a_{j_{t} j_{s}}-\left(a_{j_{t^{1}}}, \cdots, a_{j_{t} i_{k}}\right)[\boldsymbol{A}(\beta)]^{-1}\left(\begin{array}{c}
a_{i_{1} j_{s}} \\
\vdots \\
a_{i_{k} j_{s}}
\end{array}\right)\right| \\
& \geq\left|\lambda-a_{j_{t} j_{t}}\right|-\sum_{s=1, \neq t}^{l}\left|a_{j_{t} j_{s}}\right|-\sum_{s=1}^{l}\left(\left|a_{j_{t_{1}} \mid}\right|, \cdots,\left|a_{j_{t} i_{k}}\right|\right)[\mu(A(\beta))]^{-1}\left(\begin{array}{c}
\left|a_{i_{1} j_{s}}\right| \\
\vdots \\
\left|a_{i_{k} j_{s}}\right|
\end{array}\right) \\
& =\left|\lambda-a_{j_{t} j_{t}}\right|-R_{j_{t}}(A)+\sum_{v=1}^{k}\left|a_{j t_{i} i_{v}}\right|+\delta_{j_{t}}-\delta_{j_{t}}-\sum_{s=1}^{l}\left(\left|a_{j i^{i_{1}}}\right|, \cdots,\left|a_{j_{t} i_{k}}\right|\right)[\mu(\boldsymbol{A}(\beta))]^{-1}\left(\begin{array}{c}
\left|a_{i_{1} j_{s}}\right| \\
\vdots \\
\left|a_{i_{k} j_{s}}\right|
\end{array}\right) \\
& \geq\left|\lambda-a_{j_{t} j_{t}}\right|-R_{j_{t}}(A)+\delta_{j_{t}},
\end{aligned}
$$

i.e.,

$$
\left|\lambda-a_{j_{t} j_{t}}\right| \leq R_{j_{t}}(A)-\delta_{j_{t}} \leq R_{j_{t}}(A)
$$

Thus, (7) holds.

Lemma 5. [2] Let $\boldsymbol{A}=\left(a_{i j}\right) \in \mathbb{C}^{n \times n}$ and $0 \leq \alpha \leq 1$. Then for any eigenvalue $\mu$ of $\boldsymbol{A}$, there exists $1 \leq t \leq n$ such that

$$
\left|\mu-a_{t t}\right| \leq\left(R_{t}(A)\right)^{\alpha}\left(C_{t}(A)\right)^{1-\alpha} .
$$

Theorem 5. Let $\boldsymbol{A}=\left(a_{i j}\right) \in \mathbb{C}^{n \times n}, \quad \beta=\left\{i_{1}, i_{2}, \cdots, i_{k}\right\} \subseteq N_{r}(A) \cap N_{c}(\boldsymbol{A}) \neq \phi, \quad \bar{\beta}=\left\{j_{1}, j_{2}, \cdots, j_{l}\right\}, \quad 1 \leq k<n$, $\boldsymbol{A} / \beta=\left(a_{t s}^{\prime}\right)$ and $\lambda$ be eigenvalue of $\boldsymbol{A} / \beta$. Then for any $0 \leq \alpha \leq 1$, there exists $1 \leq t \leq l$ such that

$$
\left|\lambda-a_{j_{t} j_{t}}\right| \leq\left(R_{j_{t}}(\boldsymbol{A})-\delta_{t}\right)^{\alpha}\left(C_{j_{t}}(\boldsymbol{A})-\delta_{t}^{T}\right)^{1-\alpha} \leq\left(R_{j_{t}}(\boldsymbol{A})\right)^{\alpha}\left(C_{j_{t}}(\boldsymbol{A})\right)^{1-\alpha} .
$$

Proof. By Lemma 5, we know that there exists $1 \leq t \leq l$ such that

$$
\left|\lambda-a_{t t}^{\prime}\right| \leq\left(R_{t}(\boldsymbol{A} / \beta)\right)^{\alpha}\left(C_{t}(\mathrm{~A} / \beta)\right)^{1-\alpha} .
$$

Therefore,

$$
\begin{aligned}
& 0 \geq\left|\lambda-a_{t t}^{\prime}\right|-\left(R_{t}(\boldsymbol{A} / \beta)\right)^{\alpha}\left(C_{t}(\boldsymbol{A} / \beta)\right)^{1-\alpha}
\end{aligned}
$$

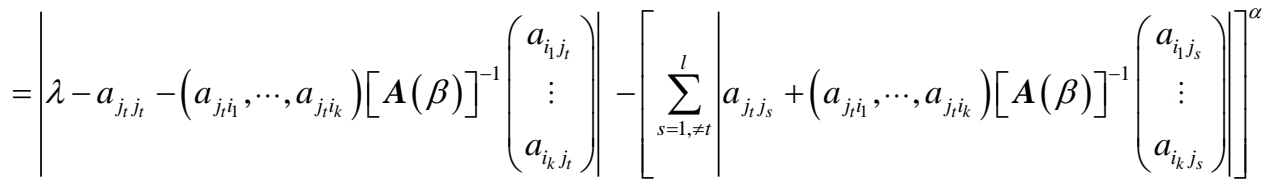

$$
\begin{aligned}
& \times\left[\sum_{s=1, \neq t}^{l}\left|a_{j_{s} j_{t}}+\left(a_{j_{s i_{1}}}, \cdots, a_{j_{s} i_{k}}\right)[\mathbf{A}(\beta)]^{-1}\left(\begin{array}{c}
a_{i_{1} j_{t}} \\
\vdots \\
a_{i_{k} j_{t}}
\end{array}\right)\right|\right]^{1-\alpha} \\
& \geq\left|\lambda-a_{j_{t} j_{t}}\right|-\left|\left(a_{j_{t_{1}} i_{1}}, \cdots, a_{j_{t} i_{k}}\right)[\boldsymbol{A}(\beta)]^{-1}\left(\begin{array}{c}
a_{i_{1} j_{t}} \\
\vdots \\
a_{i_{k} j_{t}}
\end{array}\right)\right|-\left(\sum_{s=1, \neq t}^{l}\left[\left|a_{j_{t} j_{s}}\right|+\left|\left(a_{j_{t} i_{1}}, \cdots, a_{j_{t} i_{k}}\right)[\boldsymbol{A}(\beta)]^{-1}\left(\begin{array}{c}
a_{i_{i_{1}} j_{s}} \\
\vdots \\
a_{i_{k} j_{s}}
\end{array}\right)\right|\right)^{\prime}\right. \\
& \times\left(\sum_{s=1, \neq t}^{l}\left[\left|a_{j_{s} j_{t}}\right|+\left(a_{j_{s} i_{1}}, \cdots, a_{j_{s} i_{k}}\right)[\boldsymbol{A}(\beta)]^{-1}\left(\begin{array}{c}
a_{i_{1} j_{t}} \\
\vdots \\
a_{i_{k} j_{t}}
\end{array}\right) \mid\right]\right)^{1-\alpha} .
\end{aligned}
$$


Similar as the proof of Theorem 2, we can prove

$$
\begin{aligned}
& \left|\left(a_{j_{t} i_{1}}, \cdots, a_{j_{t} i_{k}}\right)[\boldsymbol{A}(\beta)]^{-1}\left(\begin{array}{c}
a_{i_{1} j_{t}} \\
\vdots \\
a_{i_{k} j_{t}}
\end{array}\right)\right| \\
& +\left(\sum_{s=1, \neq t}^{l}\left[\left|a_{j_{t} j_{s}}\right|+\left|\left(a_{j_{t_{1}}}, \cdots, a_{j_{t} i_{k}}\right)[\boldsymbol{A}(\beta)]^{-1}\left(\begin{array}{c}
a_{i_{1} j_{s}} \\
\vdots \\
a_{i_{k} j_{s}}
\end{array}\right)\right|\right]\right)^{\alpha} \\
& \quad \times\left(\sum_{s=1, \neq t}^{l}\left[\left|a_{j_{s} j_{t}}\right|+\left|\left(a_{j_{s} i_{1}}, \cdots, a_{j_{s} i_{k}}\right)[\boldsymbol{A}(\beta)]^{-1}\left(\begin{array}{c}
a_{i_{1} j_{t}} \\
\vdots \\
a_{i_{k} j_{t}}
\end{array}\right)\right|\right]\right)^{1-\alpha} \\
& \leq\left(R_{j_{t}}(\boldsymbol{A})-\delta_{t}\right)^{\alpha}\left(C_{j_{t}}(\boldsymbol{A})-\delta_{t}^{T}\right)^{1-\alpha} .
\end{aligned}
$$

Thus, we have

$$
\begin{aligned}
0 & \geq\left|\lambda-a_{t t}^{\prime}\right|-\left(R_{t}(\boldsymbol{A} / \beta)\right)^{\alpha}\left(C_{t}(\boldsymbol{A} / \beta)\right)^{1-\alpha} \\
& \geq\left|\lambda-a_{j_{t} j_{t}}\right|-\left(R_{j_{t}}(\boldsymbol{A})-\delta_{t}\right)\left(C_{j_{t}}(\boldsymbol{A})-\delta_{t}^{T}\right)^{1-\alpha} .
\end{aligned}
$$

Further, we obtain (8).

\section{A Numerical Example}

In this section, we present a numerical example to illustrate the advantages of our derived results.

Example 1. Let

$$
\boldsymbol{A}=\left(\begin{array}{ccccc}
20 & 2 & 5 & 1 & 4 \\
2 & 15 & 2 & 4 & 1 \\
2 & 3 & 17 & 2 & 1 \\
4 & 3 & 4 & 8 & 1 \\
5 & 1 & 3 & 3 & 12
\end{array}\right), \quad \beta=\{1,3\}
$$

By calculation with Matlab 7.1, we have that

$$
\begin{gathered}
R_{1}(\boldsymbol{A})=12 ; R_{2}(\boldsymbol{A})=9 ; R_{3}(\boldsymbol{A})=8 ; R_{4}(\boldsymbol{A})=12 ; R_{5}(\boldsymbol{A})=12 ; \\
C_{1}(\boldsymbol{A})=13 ; C_{2}(\boldsymbol{A})=9 ; C_{3}(\boldsymbol{A})=14 ; C_{4}(\boldsymbol{A})=10 ; C_{5}(\boldsymbol{A})=7 ; \\
\delta_{2}=2.1800 ; \delta_{4}=4.3600 ; \delta_{5}=4.2500 ; \delta_{2}^{T}=1.4550 ; \delta_{4}^{T}=0.8404 ; \delta_{5}^{T}=1.7813 .
\end{gathered}
$$

Since $\beta \in N_{r}(\boldsymbol{A})$, by Theorem 4 , the eigenvalue inclusion set of $\boldsymbol{A} / \beta$ is

$$
\Gamma_{1}=\{\lambda|| \lambda-15 \mid \leq 6.8200\} \cup\{\lambda|| \lambda-8 \mid \leq 7.6400\} \cup\{\lambda|| \lambda-12 \mid \leq 7.7500\} .
$$

From Theorem 4 of [2], the eigenvalue inclusion set of $A / \beta$ is

$$
\Gamma_{1}^{\prime}=\{\lambda|| \lambda-15 \mid \leq 7.1412\} \cup\{\lambda|| \lambda-8 \mid \leq 8.2824\} \cup\{\lambda|| \lambda-12 \mid \leq 8.4118\} .
$$

We use Figure 1 to illustrate $\Gamma_{1} \subset \Gamma_{1}^{\prime}$. And the eigenvalues of $\boldsymbol{A} / \beta$ are denoted by “+” in Figure 1 . The blue dotted line and green dashed line denote the corresponding discs $\Gamma_{1}$ and $\Gamma_{1}^{\prime}$ respectively.

Meanwhile, since $\beta \in N_{r}(\boldsymbol{A}) \cap N_{c}(\boldsymbol{A})$, by taking $\alpha=0.5$ in Theorem 5 , the eigenvalue inclusion set of $A / \beta$ is 


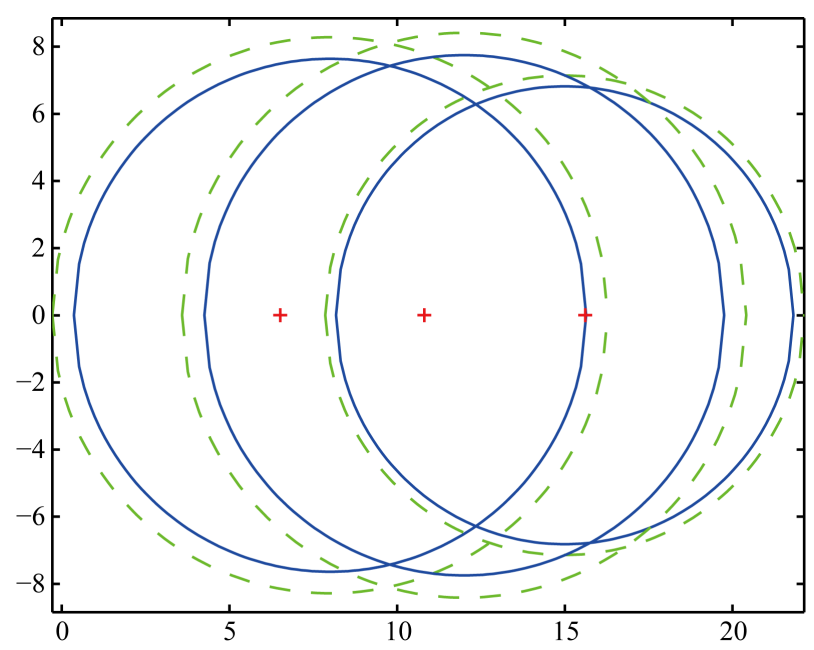

Figure 1. The blue dotted line and green dashed line denote the corresponding discs $\Gamma_{1}$ and $\Gamma_{1}^{\prime}$, respectively.

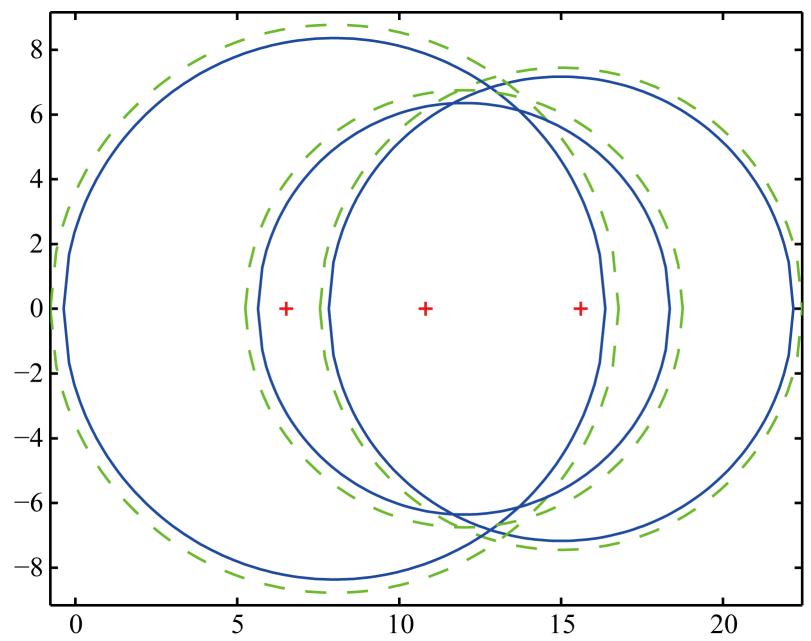

Figure 2. The blue dotted line and green dashed line denote the corresponding discs $\Gamma_{2}$ and $\Gamma_{2}^{\prime}$, respectively.

$$
\Gamma_{2}=\{\lambda|| \lambda-15 \mid \leq 7.1733\} \cup\{\lambda|| \lambda-8 \mid \leq 8.3654\} \cup\{\lambda|| \lambda-12 \mid \leq 6.3596\} .
$$

From Theorem 5 of [2], the eigenvalue inclusion set of $A / \beta$ is

$$
\Gamma_{2}^{\prime}=\{\lambda|| \lambda-15 \mid \leq 7.4492\} \cup\{\lambda|| \lambda-8 \mid \leq 8.7751\} \cup\{\lambda|| \lambda-12 \mid \leq 6.7544\} .
$$

We use Figure 2 to illustrate $\Gamma_{2} \subset \Gamma_{2}^{\prime}$. And the eigenvalues of $\boldsymbol{A} / \beta$ are denoted by “+” in Figure 2. The blue dotted line and green dashed line denote the corresponding discs $\Gamma_{2}$ and $\Gamma_{2}^{\prime}$ respectively. It is clear that $\Gamma_{1} \nsubseteq \Gamma_{2}$ and $\Gamma_{2} \nsubseteq \Gamma_{1}$.

\section{References}

[1] Cvetković, Lj. (2009) A New Subclass of $h$-Matrices. Applied Mathematics and Computation, 208, 206-210. http://dx.doi.org/10.1016/j.amc.2008.11.037

[2] Liu, J.Z. and Huang, Z.J. (2010) The Dominant Degree and Disc Theorem for the Schur Complement. Applied Mathematics and Computation, 215, 4055-4066. http://dx.doi.org/10.1016/j.amc.2009.12.063

[3] Horn, R.A. and Johnson, C.R. (1991) Topics in Matrix Analysis. Cambridge University Press, New York. 
http://dx.doi.org/10.1017/CBO9780511840371

[4] Carlson, D. and Markham, T. (1979) Schur Complements on Diagonally Dominant Matrices. Czechoslovak Mathematical Journal, 29, 246-251.

[5] Ikramov, K.D. (1989) Invariance of the Brauer Diagonal Dominance in Gaussian Elimination. Moscow University Computational Mathematics and Cybernetics, 2, 91-94.

[6] Li, B. and Tsatsomeros, M. (1997) Doubly Diagonally Dominant Matrices. Linear Algebra and Its Applications, 261, 221-235. http://dx.doi.org/10.1016/S0024-3795(96)00406-5

[7] Smith, R. (1992) Some Interlacing Properties of the Schur Complement of a Hermitian Matrix. Linear Algebra and Its Applications, 177, 137-144. http://dx.doi.org/10.1016/0024-3795(92)90321-Z

[8] Zhang, F.Z. (2005) The Schur Complement and Its Applications. Springer-Verlag, New York. http://dx.doi.org/10.1007/b105056

[9] Demmel, J.W. (1997) Applied Numerical Linear Algebra. SIAM, Philadephia.

[10] Golub, G.H. and Van Loan, C.F. (1996) Matrix Computations. 3rd Edition, Johns Hopkins University Press, Baltimore.

[11] Kress, R. (1998) Numerical Analysis. Springer, New York. http://dx.doi.org/10.1007/978-1-4612-0599-9

[12] Xiang, S.H. and Zhang, S.L. (2006) A Convergence Analysis of Block Accelerated Over-Relaxation Iterative Methods for Weak Block $H$-Matrices to Partition $\pi$. Linear Algebra and Its Applications, 418, 20-32. http://dx.doi.org/10.1016/j.laa.2006.01.013

[13] Liu, J.Z., Li, J.C., Huang, Z.H. and Kong, X. (2008) Some Properties on Schur Complement and Diagonal Schur Complement of Some Diagonally Dominant Matrices. Linear Algebra and Its Applications, 428, 1009-1030. http://dx.doi.org/10.1016/j.laa.2007.09.008

[14] Liu, J.Z. and Huang, Y.Q. (2004) The Schur Complements of Generalized Doubly Diagonally Dominant Matrices. Linear Algebra and Its Applications, 378, 231-244. http://dx.doi.org/10.1016/j.laa.2003.09.012

[15] Liu, J.Z. and Huang, Y.Q. (2004) Some Properties on Schur Complements of H-Matrices and Diagonally Dominant Matrices. Linear Algebra and Its Applications, 389, 365-380. http://dx.doi.org/10.1016/j.laa.2004.04.012

[16] Liu, J.Z. and Huang, Z.J. (2010) The Schur Complements of $\gamma$-Diagonally and Product $\gamma$-Diagonally Dominant Matrix and their Disc Separation. Linear Algebra and Its Applications, 432, 1090-1104. http://dx.doi.org/10.1016/j.laa.2009.10.021

[17] Liu, J.Z. and Zhang, F.Z. (2005) Disc Separation of the Schur Complements of Diagonally Dominant Matrices and Determinantal Bounds. SIAM Journal on Matrix Analysis and Applications, 27, 665-674. http://dx.doi.org/10.1137/040620369

[18] Li, Y.T. Ouyang, S.P. Cao, S.J. and Wang, R.W. (2010) On Diagonal-Schur Complements of Block Diagonally Dominant Matrices. Applied Mathematics and Computation, 216, 1383-1392. http://dx.doi.org/10.1016/j.amc.2010.02.038 